\title{
Scorpion Fauna of Qazvin Province, Iran (Arachnida, Scorpiones)
}

\author{
Shahrokh Navidpour* \\ Razi Reference Laboratory of Scorpion Research (RRLS), Razi Vaccine \& Serum Research Institute, \\ Agricultural Research Education and Extension Organization (AREEO), Karaj, IRAN
}

*Corresponding Author: Shahrokh Navidpour., Razi Reference Laboratory of Scorpion Research (RRLS), Razi Vaccine \& Serum Research Institute, Agricultural Research Education and Extension Organization (AREEO), Karaj, IRAN

\begin{abstract}
Six species of scorpions belonging to two families are reported from the Qazvin Province of Iran. Of these, two species are recorded from the province for the first time: Mesobuthus caucasicus (Normann, 1840) and Scorpio maurus kruglovi Birula, 1910 also presented are keys to all species of scorpions found in the Qazvin province.
\end{abstract}

ABbReViations: The institutional abbreviations listed below and used throughout are mostly after Arnett et al. (1993).

BMNH - The Natural History Museum, London, United Kingdom;

FKCP - František Kovařik Collection, Praha, Czech Republic;

MCSN - Museo Civico de Storia Naturale "Giacomo Doria”, Genova, Italy;

MHNG - Museum d'Histoire naturelle, Geneva, Switzerland;

MNHN - Muséum National d'Histoire Naturelle, Paris, France;

NHMW - Naturhistorisches Museum Wien, Vienna, Austria;

RRLS - Razi Reference Laboratory of Scorpion Research, Razi Vaccine and Serum Research Institute, Karaj, IRAN

ZISP - Zoological Institute, Russian Academy of Sciences, St. Petersburg, Russia;

ZMHB - Museum für Naturkunde der Humboldt-Universität zu Berlin, Germany;

ZMUH -Zoologisches Institut und Zoologisches Museum, Universität Hamburg, Germany.

\section{INTRODUCTION}

This paper continues a comprehensive province-by-province field study of the scorpion fauna of Iran by the RRLS team under Shahrokh Navidpour. The study includes documentation of biotope diversity, revisitation of previously known sites, some of them type localities, and sampling of all the encountered scorpion species. All specimens are collected with the help of UV light (night catch).Qazvin province is one of thirty one provinces of Iran, located in North-West of the country. The province covers $15821 \mathrm{~km}^{2}$ between $48-45$ to 50-50 east of Greenwich Meridian of longitude and 35-37 to 36-45 north latitude of the equator. The province is bounded on the north by Mazandaran and Gilan, on the west by Hamedan and Zanjan, on the south by Markazi and on the east by Tehran Provinces. The famous mountains of the province are those of Siälän, Shäh Alborz, Khashchäl, Sephidkouh, Shojä e din, Alehtareh, Rämand, Ägh dägh, Kharaghän, Saridagh, Soltan pïr, and Siähkouh, in which Siälän with a height of $4175 \mathrm{~m}$ and Shäh Alborz which is $4056 \mathrm{~m}$ are the highest. All are part of the central chain of Alborz. The lowest point of the province is in Tärom e Soflä.

The climate of the province in the northern parts is cold and snowy in winters and temperate in summers. In the southern parts the climate is mild with comparatively cold winters and warm summers. 


\section{LIST OF SCORPIONS OF QAZVIN PROVINCE}

Family Buthidae C. L. Koch, 1837

Androctonus crassicauda (Olivier, 1807)

Mesobuthus eupeus persicus (Pocock, 1899)

Mesobuthus caucasicus (Normann, 1840)

Odontobuthus doriae (Thorell, 1876)

Orthochirus zagrosensis kovarik, 2004

Family Scorpionidae Latreille, 1802

Scorpio maurus kruglovi Birula, 1910 (first report)

Systematics

Family Buthidae C. L. Koch, 1837

Androctonus crassicauda (Olivier, 1807)

Scorpio crassicauda Olivier, 1807: 97.

Buthus crassicauda: Simon, 1872: 247 (in part); Simon, 1879: 99; Simon, 1892: 83; Kraepelin, 1899: 16; Pocock, 1902: 373; Kraepelin, 1913: 124; Lampe, 1918: 190.

Androctonus crassicauda: Kraepelin, 1891: 175 (in part); Vachon, 1951: 343; Khalaf, 1962: 1; Khalaf, 1963: 60; Habibi, 1971: 42; Farzanpay \& Pretzmann, 1974: 215; Pérez Minocci, 1974: 17; Vachon, 1974: 909; Vachon, 1979: 31; Farzanpay, 1987: 141; Farzanpay, 1988: 36; Fet, 1989: 78; AlSafadi, 1992: 96; Amr \& El-Oran, 1994: 187; Sissom, 1994: 36; Dupré et al., 1998: 59; Kovařík, 1998: 104; Crucitti, 1999: 83; Kabakibi et al., 1999: 80; Fet \& Lowe, 2000: 72; Stathi \& Mylonas, 2001: 288; Crucitti \& Vignoli, 2002: 439; Kovař́k, 2002: 5; Fet \& Kovař́k, 2003: 180; Vignoli et al., 2003: 2; Kovařík \& Whitman, 2005: 105; Lourenço, 2005: 149; Hendrixson, 2006: 38; Akbari, 2007: 76, fig. p. 62; Navidpour et al., 2008a:; Navidpour et al., 2008b: 3; Navidpour et al., 2008c: 3; Navidpour et al., 2008d: 3; Pirali-Kheirabadi et al., 2009: 3; Navidpour et al., 2010: 3; Navidpour et al., 2011: 3; Navidpour et al., 2012: 3.

Prionurus crassicauda: Pocock, 1895: 292; Tullgren, 1909: 2; Birula, 1904: 29; Birula, 1905a: 120; Masi, 1912: 91; Penther, 1912: 110.

Androctonus crassicauda crassicauda: Vachon, 1959: 124; Vachon, 1966: 210; Habibi, 1971: 42; Vachon, 1979: 34; Levy \& Amitai, 1980: 23-29; Kovařík, 1997: 49.

= Prionurus crassicauda orientalis Birula, 1900: 355; Birula, 1903: 67 (syn. by Fet, 1989: 79).

Buthus (Prionurus) crassicauda orientalis: Birula, 1917: 93, 240.

Buthus crassicauda orientalis: Kraepelin, 1913: 124.

Androctonus crassicauda orientalis: Vachon, 1959: 124; Vachon, 1966: 210; Habibi, 1971: 42; Pérez Minocci, 1974: 18.

Androctonus amoreuxi baluchicus: Kovařík, 1997: 39 (see Vignoli et al., 2003: 4).

TYPE LOCALITY AND TYPE REPOSITORY. Kashan, Persia, now Iran, Esfahan Province; MNHN.

QAZVIN Province Material EXAMINED. Iran, Qazvin Province, Zitak village, 36 31'32.2"N 49 44'49.6"E, $800 \mathrm{~m}$ a.s.l. (Locality No. Q-102), V.2015, $7 \lesssim 3$ ㅇ (RRLS), leg. Nikoonam; Looshan, 360'19.6"N 49²4'22.3"E, 860 m a.s.l. (Locality No. Q-106), V.2015, 4 3 우 (RRLS), leg. Nikoonam; Abyek, 3605'68.2"N 5053'58.6"E, $1008 \mathrm{~m}$ a.s.l. (Locality No. Q-107), V.2015, $10^{\Uparrow}$ (RRLS), leg. Nikoonam; Ardagh village, 3604'07.6"N 4945'37.3"E, $1241 \mathrm{~m}$ a.s.l. (Locality No. Q109), V.2015, 2^1우 (RRLS), leg. Nikoonam; Booein Zahra, 3547'39.2"N 5002'39.6"E, $1210 \mathrm{~m}$ a.s.1. (Locality No. Q-112), V.2015, 10 (RRLS), leg. Nikoonam; Heer village, 36 59'54.6"N 50²5'52.3"E, 830 m a.s.l. (Locality No. Q-120), V.2015,2今 (RRLS), leg. Nikoonam.

DISTRIBUTION: Widespread in Iran, found in most provinces. Recorded also from Armenia (Kraepelin, 1899: 17), Azerbaijan (Fet, 1989: 79), Bahrain (Crucitti \& Vignoli, 2002: 439), Egypt (Fet \& Lowe, 2000: 72), Iraq (Kennedy, 1937: 745), Israel (Simon, 1892: 83), Jordan (Amr \& El-Oran, 1994: 187), Kuwait (Kettel, 1982: 6), Lebanon (El-Hennawy, 1992: 100), Oman (Birula, 1917: 229; Hendrixson, 2006: 39), Qatar (El-Hennawy, 1992: 100), Saudi Arabia (Pocock, 1895: 292; Hendrixson, 2006: 39), Syria (Simon, 1872: 247), Tunisia (Kraepelin, 1901: 266), Turkey (Pocock, 1902: 373), United Arab Emirates (Hendrixson, 2006: 40), and Yemen (Birula, 1937: 101). 


\section{MESOBUTHUS CAUCASICUS (NORMANN, 1840)}

QAZVIN Province Material EXAMINED. Iran, Qazvin Province, Zitak village, 36 31'32.2"N 4944'49.6"E, 800 m a.s.l. (Locality No. Q-102), V.2015, 5ð1우 (RRLS), leg. Nikoonam.

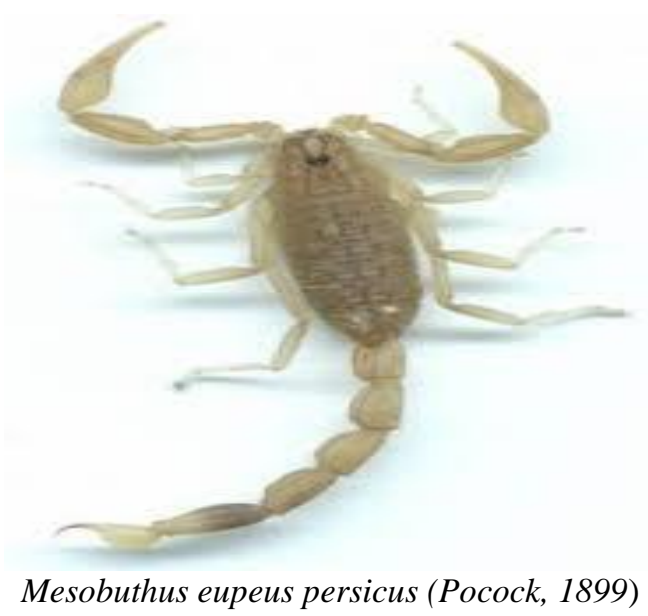

Buthus caucasicus persicus Pocock, 1899: 404; Pocock, 1900: 19.

Buthus eupeus persicus: Birula, 1905a: 119, 122, 124-126; Birula, 1918: 10-13, figs.

Mesobuthus eupeus persicus: Vachon, 1959: 155; Vachon, 1966: 212; Habibi, 1971: 44; Farzanpay, 1988: 38; Fet, 1994: 527; Kovař́k, 1997: 49; Kovařík, 1998: 114; Fet \& Lowe, 2000: 174; Navidpour et al., 2011: 9, 6, 12, 41-44; Navidpour et al., 2012: 10.

Mesobuthus caucasicus persicus: Pérez Minocci, 1974: 25; Capes \& Fet, 2001: 303.

= ? Buthus eupeus kirmanensis Birula, 1900: 364 (see Fet \& Lowe, 2000: 173).

Buthus (Buthus) eupeus kirmanensis: Birula, 1917: 239; Birula, 1918: 14.

Mesobuthus eupeus kirmanensis: Vachon, 1959: 155; Vachon, 1966: 212; Habibi, 1971: 43; Farzanpay, 1988: 38; Fet, 1994: 527; Kovař́k, 1997: 49; Kovařík, 1998: 114; Fet \& Lowe, 2000: 173; Mirshamsi et al., 2011: 9.

= ? Buthus pachysoma Birula, 1900: 370 (syn. by Navidpour et al., 2011: 9).

Buthus eupeus pachysoma: Birula, 1905a: 128.

Buthus (Buthus) eupeus pachysoma: Birula, 1917: 239.

Mesobuthus eupeus pachysoma: Vachon, 1959: 155; Vachon, 1966: 212; Habibi, 1971: 43; Farzanpay, 1988: 38; Fet, 1994: 527; Kovařík, 1997: 49; Kovařík, 1998: 114; Fet \& Lowe, 2000: 174.

TYPE LOCAliTY AND TYPE REPOSITORY. Persia, now Iran, East Azarbaijan, Seir, E side of Lake Urmia (Urmi); BMNH.

TYPE MATERIAL EXAMINED. Iran, East Azarbaijan Province, Seir, Lake Urmi, $1 q$ lectotype, BMNH No. 1900.1,15.

QAZVIN Province MATERIAL EXAMINED. Iran, Qazvin Province, Takestan, 36 $53^{\prime} 47.2^{\prime \prime} \mathrm{N}$ 49 38'52.6"E, $1265 \mathrm{~m}$ a.s.l. (Locality No. Q-101), V.2015, 22ð13ᄋ (RRLS), leg. Nikoonam; Zitak village, $36^{\circ} 31^{\prime} 32.2^{\prime \prime} \mathrm{N} 49^{\circ} 44^{\prime} 49.6 " \mathrm{E}, 800 \mathrm{~m}$ a.s.1. (Locality No. Q-102), V.2015, $7 \jmath^{\Uparrow} 3$ + (RRLS), leg. Nikoonam; Bon Alamoot, 36³9'57.2"N 5057'52.6"E, 1265 m a.s.l. (Locality No. Q-103), V.2015,

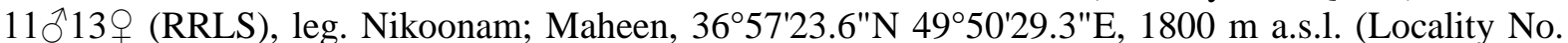
Q-104), V.2015, 24 8 \% (RRLS), leg. Nikoonam; Siyahpoosh, 36 $56^{\prime} 44.2^{\prime \prime N} 49^{\circ} 34^{\prime} 43.6^{\prime \prime E}, 1050 \mathrm{~m}$ a.s.l. (Locality No. Q-105), V.2015, 31 111 ( 49 17'13.3"E, $1153 \mathrm{~m}$ a.s.l. (Locality No. Q-108), V.2015, 7ð 1 ㅇ (RRLS), leg. Nikoonam; Ardagh village, $36^{\circ} 04^{\prime} 07.6^{\prime \prime} \mathrm{N} 49^{\circ} 45^{\prime} 37.3^{\prime \prime E}, 1241 \mathrm{~m}$ a.s.l. (Locality No. Q-109), V.2015, $12{ }^{\top} 6$ 우 (RRLS),

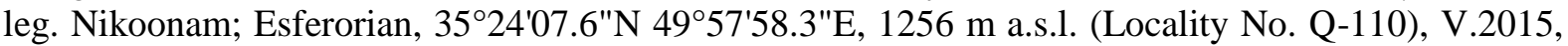
$11 \delta^{\wedge} 8$ 우 (RRLS), leg. Nikoonam; Eghbaliyeh, 36 22'56.6"N 4955'57.3"E, $1256 \mathrm{~m}$ a.s.l. (Locality 
No. Q-109), V.2015, 2`25ㅇ (RRLS), leg. Nikoonam; Booein Zahra, 3547'39.2"N 5002'39.6"E, $1210 \mathrm{~m}$ a.s.1. (Locality No. Q-112), V.2015, $18{ }^{\lambda} 6$ ㅇ (RRLS), leg. Nikoonam; Heer village, 36 59'54.6"N 50²5'52.3"E, $830 \mathrm{~m}$ a.s.1. (Locality No. Q-120), V.2015, 19 ${ }^{\Uparrow} 4$ 우 (RRLS), leg. Nikoonam; Roodbar Alamoot, 36 57'39.2"N 5006'59.6"E, $910 \mathrm{~m}$ a.s.l. (Locality No. Q-121), V.2015, 401우 (RRLS), leg. Nikoonam; Changoor village, 36²3'56.6"N 4948'34.3"E, $810 \mathrm{~m}$ a.s.1.

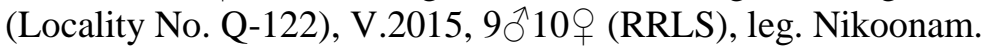

DisTRIBUTION: Iran, East Azarbaijan (Pocock, 1899: 404), Fars (Navidpour et al., 2012: 11), Kerman, Sistan \& Baluchistan (Birula, 1900: 364; Fet \& Lowe, 2000: 173), Hormozgan (first report), and Yazd (Mirshamsi et al., 2011: 9) Provinces; Pakistan (Pocock, 1900: 20).

\section{ODONTOBUTHUS DORIAE (THORELL, 1876)}

Buthus doriae Thorell, 1876: 107; Kraepelin, 1891: 57-58, pl. I; Kraepelin, 1899: 27.

Odontobuthus doriae: Pringle, 1960: 83; Khalaf, 1963: 66; Vachon, 1966: 213; Habibi, 1971: 44; Pérez Minocci, 1974: 28; Farzanpay, 1988: 39; Kovařík, 1997: 47; Kovařík, 1998: 115; Fet \& Lowe, 2000: 187; Kovař́ík, 2002: 9; Lourenço \& Pézier, 2002: 116; Vignoli et al., 2003: 4; Pirali-Kheirabadi et al., 2009: 9; Navidpour et al., 2011: 13; Karatas et al., 2012: 116; Navidpour et al., 2012: 14.

TYPE LOCALITY AND TYPE REPOSITORY. Iran, Teheran; MCSN.

QAZVIN Province MATERIAL EXAMINED. Iran, Qazvin Province, Zitak village, 36 31'32.2"N 49 44'49.6"E, $800 \mathrm{~m}$ a.s.1. (Locality No. Q-102), V.2015, 201ㅇ (RRLS), leg. Nikoonam;

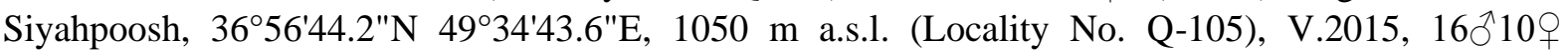
(RRLS), leg. Nikoonam; Abyek, 3605'68.2"N 5053'58.6"E, $1008 \mathrm{~m}$ a.s.l. (Locality No. Q-107), V.2015, 9 912 ㅇ (RRLS), leg. Nikoonam; Avaj village, 3554'48.6"N 49¹7'13.3"E, $1153 \mathrm{~m}$ a.s.l. (Locality No. Q-108), V.2015, 6万2우 (RRLS), leg. Nikoonam; Ardagh village, 36 04'07.6"N 4945'37.3"E, 1241 m a.s.1. (Locality No. Q-109), V.2015, 1ð2우 (RRLS), leg. Nikoonam.

DiSTRIBUTION: Iran, Esfahan, Fars, Hamadan, Kerman, Kermanshah, Mazandaran, Markazi, Teheran, West Azarbaijan, Yazd (Kovařík, 1997: 47, Lourenço \& Pézier, 2002: 116, 117, 124), Chahar Machal \& Bakhtiyari (Pirali-Kheirabadi et al., 2009: 10), Tehran, and Sistan \& Baluchistan (Pirali-Kheirabadi et al., 2009: 14) Provinces.

\section{Orthochirus Zagrosensis Kovařík, 2004}

Simonoides farzanpayi Vachon \& Farzanpay in Farzanpay, 1987: 162; Farzanpay, 1988: 41; Fet \& Lowe, 2000: 223.

Orthochirus farzanpayi Kovař́k \& Fet, 2006a: 1; Navidpour et al., 2008a: 14; Navidpour et al., 2008b; Navidpour et al., 2011: 15; Navidpour et al., 2012: 16.

= Orthochirus sobotniki Kovař́k, 2004: 20 (syn. by Kovařík \& Fet, 2006a: 1).

= Paraorthochirus goyffoni Lourenco \& Vachon, 1995: 301; Fet \& Lowe, 2000: 212. Syn. n.

TYPE LOCALITY AND TYPE REPOSITORY. Iran, 215 km N of Bandar-e-Abbas; NHMW.

TYPE MATERIAL EXAMINED. Iran, $215 \mathrm{~km} \mathrm{~N}$ of Bandar-e-Abbas, 22.III.1972, 1 q (lectotype) $1 \mathrm{\jmath}^{1} 1$ q (paralectotypes) (NHMW Nos. 68-70, rev. Max Vachon in 1977, No. VA 1910); $5 \mathrm{~km} \mathrm{SE} \mathrm{of} \mathrm{Posht}$

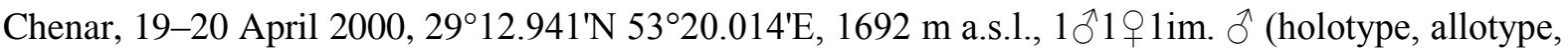
and paratype of Orthochirus sobotniki) (FKCP), leg. J. Šobotník.

TYPE LOCALITY AND TYPE REPOSITORY. Iran, Dasht-E-Arzhan, 29³4'644"N 5156'889"E, alt. $2000 \mathrm{~m}$; FKCP.

QAZVIN Province MATERIAL EXAMINed. Iran, Qazvin Province, Zitak village, 36 31'32.2"N 4944'49.6"E, 800 m a.s.1. (Locality No. Q-102), V.2015, $7{ }^{\Uparrow} 1$ ㅇ (RRLS), leg. Nikoonam.

DisTRIBUTION: Iran, Hormozgan, Fars (Kovařík \& Fet, 2006a: 1-3), Khoozestan (Navidpour et al., 2008a: 15), Bushehr (Navidpour et al., 2008b: 15), Tehran (first report) and Kerman (Navidpour et.

al2011: 15) Province 


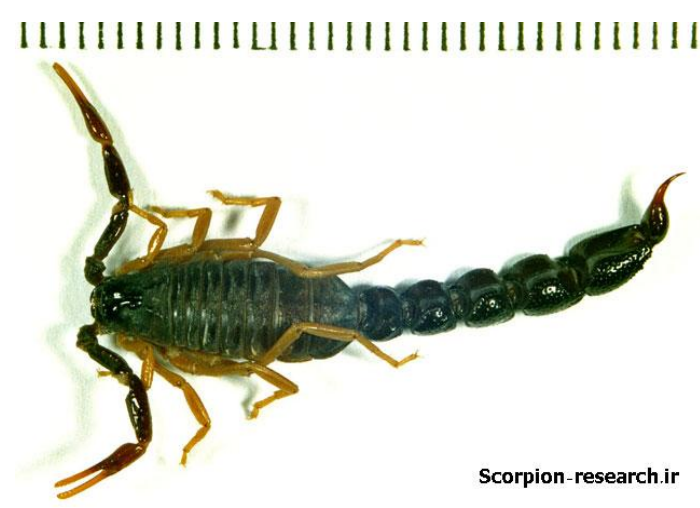

Family Scorpionidae Latreille, 1802

Scorpio maurus kruglovi Birula, 1910

Heterometrus townsendi Pocock, 1900: 364.

? Scorpio townsendi: Birula, 1905a: 147 (Birula, 1910: 184).

Scorpio maurus townsendi: Birula, 1910: 184.

Scorpio maurus: Farzanpay, 1987: 165; Farzanpay, 1988: 42; Akbari, 2007: 76.

TYPE LOCALITY AND TYPE REPOSITORY. Iran, Bushehr Province, Fort Reshire near Bushire, Persian Gulf, Iran; BMNH

QAZVIN PROVINCE MATERIAL EXAMINED. Iran, Qazvin Province,Takestan, 36 53'47.2"N 49 38'52.6"E, 1265 m a.s.l. (Locality No. Q-101), V.2015, $10{ }^{7} 8$ ㅇ (RRLS), leg. Nikoonam; Looshan, $36^{\circ} 40^{\prime} 19.6 " \mathrm{~N} 49^{\circ} 24^{\prime 22} .3 " \mathrm{E}, 860 \mathrm{~m}$ a.s.1. (Locality No. Q-106), V.2015, 6○12우 (RRLS), leg. Nikoonam.

DisTRIBUTION: DistRIBUTION: Iraq, Jordan, Kuwait, Qatar, Saudi Arabia, Syria (Kinzelbach, 1985; Pringle, 1960: 86; Vachon, 1979: 57), Iran (Hamadan, Khermanshah, North Khorasan, Mazendran, Qazvin, Semnan, Tehran (Karatas et al., 2012), Alborz and Markazi provinces and Qazvin Province (first report).

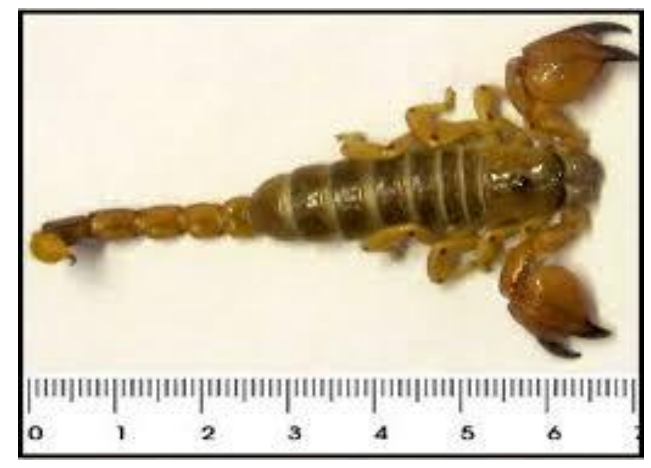

\section{ACKNOWLEDGMENT}

This study was financially supported by the Razi Vaccine \& Rerum Research Institute of Iran. We thank Behzad Masihipour, Falahpoor, Barzegar, Rabiei, Habibzadeh, Behmam and Bahrani for their valu-able contributions, and all those who helped us in collecting samples. We also thank two anonymous reviewers for their comments

\section{REFERENCES}

[1] AL-SAFADI, M. M. 1992. Additions to the scorpion fauna of Yemen. Zoology in the Middle East, 6: 95-99.

[2] AMR, Z. S. \& R. EL-ORAN. 1994. Systematics and distribution of scorpions (Arachnida, Scorpionida) in Jordan. Bolletino di Zoologia, 61(2): 185-190.

[3] ARNETT, H. R. JR., G. A. SAMUELSON \& G. M. NISHIDA. 1993. The Insect and Spider Collections of the World. Flora \& Fauna Handbook No. 11, Second edition. Gainesville: Sandhill Crane Press, 308 pp. 
[4] AUSSERER, A. 1880. Arachnida. Zoologischer Jahresbericht, 1879: 430-470.

[5] BIRULA, A. A. 1900. Beiträge zur Kenntniss der Scorpionenfauna Ost-Persiens. Bulletin de l'Académie Impériale des Sciences de St.-Pétersbourg, 12(1): 355-375.

[6] BIRULA, A. A. 1903. Beiträge zur Kenntniss der Scorpionenfauna Persiens (Zweiter Beiträg). Bulletin de l'Académie Impériale des Sciences de St.-Pétersbourg, 19: 67-80.

[7] BIRULA, A. A. 1904. Miscellanea scorpiologica. VII. Synopsis der russischen Skorpione. Annuaire du Musée Zoologique de l'Académie Impériale des Sciences de St.-Pétersbourg, 9: 28-38.

[8] BIRULA, A. A. 1905a. Beiträge zur Kenntniss der Scorpionenfauna Persiens (Dritter Beiträge). Bulletin de l'Académie Impériale des Sciences de St.-Pétersbourg, 23: 119-148.

[9] BIRULA, A. A. 1905b. 4. Skorpiologische Beiträge, 1.-3. Microbuthus littoralis (Pavesi), Anomalobuthus rickmersi Kraepelin und Buthus zarudnianus n. nom. Zoologischer Anzeiger, 29(14): 445-450.

[10] (BIRULA, A. A.) BYALYNITSKII-BIRULYA, A. A. 1917. Arachnoidea Arthrogastra Caucasica. Pars I. Scorpiones. Zapiski Kavkazskogo Muzeya (Mémoires du Musée du Caucase), Tiflis: Imprimerie de la Chancellerie du Comité pour la Transcaucasie, A(5), 253 pp. (in Russian; published August 1917). English translation: Byalynitskii-Birulya, A. A. 1964. Arthrogastric Arachnids of Caucasia. 1. Scorpions. Jerusalem: Israel Program for Scientific Translations, 170 pp. (in Russian).

[11]BIRULA, A. A. 1918. Miscellanea scorpiologica. XI. Materialy k skorpiofaune nizhnei Mesopotamii, Kurdistana i Severnoi Persii (Matériaux pour servir á la scorpiofaune de la Mésopotamie inférieure, du Kurdistan et de la Perse septentrionale). Annuaire du Musée Zoologique de l'Académie Impériale des Sciences de St.-Pétersbourg, 22(1917): 1-44 (in Russian).

[12] CRUCITTI, P. 1999. The scorpions of Anatolia: biogeographical patterns. Biogeographia, 20: 81-94.

[13] CRUCITTI, P. \& V. VIGNOLI. 2002. Gli Scorpioni (Scorpiones) dell'Anatolia sud-orientale (Turchia). Bolletino della Museo Scienze Naturali in Torino, 19(2): 433-474.

[14] DUPRÉ, G., N. LAMBERT \& P. GÉRARD. 1998. Les Scorpions. Biologie. Élevage. Paris, 82 pp.

[15] EL-HENNAWY, H. K. 1992. A catalogue of the scorpions described from the Arab countries (1758-1990) (Arachnida: Scorpionida). Serket, 2(4): 95-153.

[16]FARZANPAY, R. 1986. Mesobuthus eupeus, an indigenous scorpion from Iran. Origin and its geographical distribution. In Barrientos, J.A. (ed.), Actas X. Congreso Internacional de Aracnologia. Jaca (Espaňa) Septiembre 1986, 1: 333-335.

[17]FARZANPAY, R. 1987 (1366). [Knowing Scorpions]. Teheran: Central University Publications, No. 312 , Biology 4, 231 pp. (in Farsi, with Latin index).

[18]FARZANPAY, R. 1988. A catalogue of the scorpion's occuring in Iran, up to January 1986. Revue Arachnologique, 8(2): 33-44.

[19]FARZANPAY, R. \& G. PRETZMANN. 1974. Ergebnisse einiger Sammelreisen nach Vorderasien 4. Teil: Skorpione aus Iran. Annalen des Naturhistorischen Museuns in Wien, 78: 215-217.

[20]FET, V. 2000. Family Scorpionidae Latreille, 1802. Pp. 427-486 in Fet, V., Sissom, W. D., G. Lowe \& M. E. Braunwalder. 2000. Catalog of the Scorpions of the World (1758-1998). The New York Entomological Society, New York, 689 pp.

[21] HEMPRICH, F. G. \& CH. G. EHRENBERG 1831. Animalia Articulata, Arachnoidea, Scorpiones. In: Symbolae physicae animalia evertebrata, exclusis insectis, series prima, tabularum decade prima. Berolini: Officina Academica 12 pp.

[22] HENDRIXSON, B. E. 2006. Buthid scorpions of Saudi Arabia, with notes on other families (Scorpiones: Buthidae, Liochelidae, Scorpionidae). Fauna of Arabia, 21: 33-120.

[23] KABAKIBI, M. M., N. KHALIL \& Z. AMR. 1999. Scorpions of southern Syria. Zoology in the Middle East, 17: 79-89.

[24] KARATAŞ A., M. M. GARKHELOO \& M. UÇAK 2012. Contribution to the distribution of the scorpions of Iran. Zoology in the Middle East, 55: 111-120.

[25] KHALAF, L. 1962. A small collection of scorpions from Iraq. Bulletin of the Iraq Natural History Institute, 2(4): 1-3.

[26] KHALAF, K. I. 1963. Scorpions reported from Iraq. Bulletin of Endemic Diseases (Baghdad), 5(1-2): 59-70.

[27] KOVAŘÍK, F. 1992. A check list of scorpions (Arachnida: Scorpiones) in the collections of the Zoological Depertment, National Museum in Prague. Acta Societatis Zoologicae Bohemoslovaca, 56: 181-186.

[28]KOVAŘÍK, F. 1997. Results of the Czech Biological Expedition to Iran. Part 2. Arachnida: Scorpiones with descriptions of Iranobuthus krali gen. n. et sp. n. and Hottentotta zagrosensis sp. n. (Buthidae). Acta Societatis Zoologicae Bohemicae, 61: 39-52. 
[29]KOVAŘÍK, F. 2007. A revision of the genus Hottentotta Birula, 1908, with descriptions of four new species (Scorpiones, Buthidae). Euscorpius, 58: 1-107.

[30]KOVAŘÍK, F. 2009. Illustrated catalog of scorpions. Part I. Introductory remarks; keys to families and genera; subfamily Scorpioninae with keys to Heterometrus and Pandinus species. Clairon Production, Prague, $170 \mathrm{pp}$.

[31] KOVAŘÍK F. \& Z. AHMED. 2007. Two new species of the genus Compsobuthus Vachon, 1949 from Afghanistan and Pakistan (Scorpiones: Buthidae). Euscorpius, 53: 1-6.

[32] KOVAŘÍK, F \& A. A. OJANGUREN AFFILASTRO. 2013. Illustrated catalog of scorpions Part II. Bothriuridae; Chaerilidae; Buthidae I., genera Compsobuthus, Hottentotta, Isometrus, Lychas, and Sassanidotus. Clairon Production, Prague, xy pp.

[33] KOVǍ̌íK, F., E. A. YAĞMUR, V. FET \& S. NAVIDPOUR 2011. On two subspecies of Mesobuthus eupeus (C. L. Koch, 1839) in Turkey (Scorpiones: Buthidae). Euscorpius, 109: 1-15.

[34] KRAEPELIN, K. 1891. Revision der Skorpione. I. Die Familie des Androctonidae. Jahrbuch der Hamburgischen Wissenschaftlichen Anstalten, 8(1890): 144-286 (1-144).

[35] KRAEPELIN, K. 1895. Nachtrag zu Theil I der Revision der Skorpione. Jahrbuch der Hamburgischen Wissenschaftlichen Anstalten, 12(1894): 73-96.

[36] KRAEPELIN, K. 1901. Catalogue des Scorpions des collections du Muséum d'Histoire Naturelle de Paris. Bulletin du Muséum national d'Histoire naturelle, Paris, 7: 265-274.

[37]LOURENÇO, W. R. 1996. A new genus and a new species of scorpion (Buthidae) from Iran. Zoology in the Middle East, 12: 93-98.

[38] LOURENÇO, W. R. 2005. Nouvelles considérations taxonomiques sur les espèces du genre Androctonus Ehrenberg, 1828 et description de deux nouvelles espèces (Scorpiones, Buthidae). Revue suisse de Zoologie, 112 (1) : 145-171

[39]LOURENÇO, W. R. \& D. HUBER 2000. Two new species of scorpions (Scorpiones, Buthidae) of the genus Paraorthochirus Lourenço \& Vachon from Oman. Zoology in the Middle East, 20: 137-144.

[40] LOURENÇO, W. R. \& A. PÉZIER. 2002. Taxonomic consideration of the genus Odontobuthus Vachon (Scorpiones, Buthidae), with descripion of a new species. Revue suisse de Zoologie, 109(1): 115-125.

[41]LOWE, G. 2010. A new species of Odontobuthus (Scorpiones: Buthidae) from northern Oman. Euscorpius, 96: $1-22$.

[42] MIRSHAMSI, O., A. SARI, E. ELAHI \& S. HOSSEINIE 2011. Mesobuthus eupeus (Scorpiones: Buthidae) from Iran: a polytypic species complex. Zootaxa, 2929: 1-21.

[43] NAVIDPOUR, S., F. KOVǍ̌íK, M. E. SOLEGLAD \& V. FET. 2008a. Scorpions of Iran (Arachnida, Scorpiones). Part I. Khoozestan Province. Euscorpius, 65: 1-41.

[44] NAVIDPOUR, S., M. E. SOLEGLAD, V. FET \& F. KOVAŘÍK, 2008b. Scorpions of Iran (Arachnida, Scorpiones). Part II. Bushehr Province. Euscorpius, 67: 1-33.

[45] NAVIDPOUR, S., V. FET, F. KOVAǨÍK \& M. E. SOLEGLAD, 2008c. Scorpions of Iran (Arachnida, Scorpiones). Part III. Ilam Province. Euscorpius, 69: 1-29.

[46] NAVIDPOUR, S., F. KOVAĽÍK, M. E. SOLEGLAD \& V. FET. 2008d. Scorpions of Iran (Arachnida, Scorpiones). Part IV. Kohgilouyeh \& Boyer Ahmad Province. Euscorpius, 74: 1-24.

[47] NAVIDPOUR, S., H. H. NAYEBZADEH, M. E. SOLEGLAD, V. FET, F. KOVAŘÍK \& M. H. KAYEDI. 2010. Scorpions of Iran (Arachnida, Scorpiones). Part VI. Lorestan Province. Euscorpius, 99: 1-23.

[48] NAVIDPOUR, S., M. EZATKHAH, F. KOVAŘIK, M. E. SOLEGLAD \& V. FET 2011. Scorpions of Iran (Arachnida, Scorpiones). Part VII. Kerman Province. Euscorpius, 131: 1-32.

[49] NAVIDPOUR, S., V. FET, F. KOVAŘIK \& M. E. SOLEGLAD 2012. Scorpions of Iran (Arachnida, Scorpiones). Part VIII. Fars Province. Euscorpius, 139: 1-29.

[50] OLIVIER, G. A. 1807. Voyage dans l'Empire Othoman, l'Égypte et la Perse. Henri Agasse, Paris, Vol. 3: 96-97, fig. 2.

[51]PETERS, W. 1861a. Eine neue Untergattung von Scorpionen, Hemiscorpion lepturus. Monatsberichte der Königlichen Preussischen Akademie der Wissenschaften zu Berlin, 1861: 426-427.

[52] PETERS, W. 1861b. Über eine neue Eintheilung der Skorpione und über die von ihm in Mossambique gesammelten Arten von Skorpionen. Monatsberichte der Königlichen Preussischen Akademie der Wissenschaften zu Berlin, 1861: 507-520.

[53] PIRALI-KHEIRABADI, K., S. NAVIDPOUR, V. FET, F. KOVAŘÍK \& M. E. SOLEGLAD. 2009. Scorpions of Iran (Arachnida, Scorpiones). Part V. Chahar Mahal \& Bakhtiyari Province. Euscorpius, 78: 1-23.

[54]POCOCK, R. I. 1889. Notes on some Buthidae, new and old. Annals and Magazine of Natural History, 6(3): 334-351. 
[55] POCOCK, R. I. 1895. On the Arachnida and Myriapoda obtained by Dr. Anderson`s collector during Mr. T. Bent's expedition to the Hadramaut, South Arabia; with a supplement upon the scorpions obtained by Dr. Anderson in Egypt and the Eastern Soudan. Journal of the Linnaean Society, 25: 292-316.

[56] POCOCK, R. I. 1899. Chilopoda and Arachnida. In R. T. Günther. Contributions to the natural history of Lake Urmi, N. W. Persia, and its neighbourhood. Journal of the Linneaen Society of London, Zoology, 27: 399-406.

[57] SIMON, E. 1872. Arachnides de Syrie, rapportés par M. Charles Piochard de la Brulerie (Scorpions et Galéodes). Annales de la Sociéte Entomologique de France, (5)2: 245-266.

[58] SIMON, E. 1879. 3e Ordre. Scorpiones. Pp. 79-115 in : Les Arachnides de France. VII. Contenant les Ordres des Chernetes, Scorpiones et Opiliones. Paris: Roret.

[59] SIMON, E. 1880a. Études Arachnologiques 12e Mémoire. Part XVIII. Descriptions de Genres et Espéces de l’orde des Scorpiones. Annales de la Sociéte Entomologique de France, 5(10)1880: 377-398.

[60] SIMON, E. 1880b. Quelques scorpions qui lui ont été donnés par notre confrére M. Reiche, de la part de M. F. de Saulcy, qui les a recus de Mossoul (ancienne Ninive), sur le Tigre, en Mésopotamie. Annales de la Sociéte Entomologique de France, 5(10): 29.

[61] SIMON, E. 1892. Liste des Arachnides Recueillis en Syrie par M. le Dr Théod. Barrois. Revue Biologique du Nord de la France, 5: 80-84.

[62] SISSOM, W. D. 1990. Systematics, biogeography and paleontology. Pp. 64-160 in Polis, G. A. (ed.), The Biology of Scorpions. Stanford: Stanford University Press, $587 \mathrm{pp}$.

[63] VACHON, M. 1940. Sur la systématique des scorpions. Mémoires du Muséum National d'Histoire Naturelle, Paris, 13(2): 241-259.

[64] VACHON, M. 1951. Prof. Kosswig tarafindan Türkiyede toplanan akrepler hakkinda. Á propos de quelques Scorpions de Turquie collectés par M. le Professeur Dr. Curt Kosswig. Revue de la Faculté des Sciences de l'Université d'Istanbul, ser. B, 16(4): 341-344.

[65] VACHON, M. 1952. Études sur les Scorpions. Institut Pasteur d’Algérie, Alger, 482 pp. (published 19481951 in Archives de l'Institut Pasteur d'Algérie, 1948, 26: 25-90, 162-208, 288-316, 441-481. 1949, 27 : 66-100, 134-169, 281-288, 334-396. 1950, 28: 152-216, 383-413. 1951, 29: 46-104).

[66] VACHON, M. 1959. Scorpionidea (Chelicerata) de l'Afganistan. The 3rd Danish Expedition to central Asia (Zoological Results 23). Videnskabelige Meddelelser fra Dansk Naturhistorisk Forening i Kobehavn, 120: 121-187.

[67] VACHON, M. 1979. Arachnids of Saudi Arabia, Scorpiones. Fauna Saudi Arabia 1: 30-66.

[68] VACHON, M. \& R. KINZELBACH. 1987. On the taxonomy and distribution of the scorpions of the Middle East. In Krupp, F., W. Schneider \& R. Kinzelbach (eds.), Proceedings of the Symposium on the Fauna and Zoogeography of the Middle East, Mainz (TAVO), 28(1985): 91-103.

[69] VACHON, M. \& R. STOCKMANN. 1968. Contribution á létude des Scorpions africains appartenant au genre Buthotus Vachon 1949 et étude de la variabilité. Monitore Zoologico Italiano, Firenze 1968 (2. Supplemento): 81-149.

[70] VIGNOLI, V. 2005. Description of a new species of Compsobuthus Vachon, 1949 (Scorpiones: Buthidae) from southern Iran. Zoology in the Middle East, 34: 79-86.

[71]WEIDNER, H. 1959. Die entomologischen Sammlungen des Zoologischen Staatsinstituts und Zoologischen Museums Hamburg, I. Teil, Pararthropoda und Chelicerata I. Mitteilungen aus dem Hamburgischen Zoologischen Museum und Institut, 57: 89-142.

[72] YAĞMUR, E. A, M. YALCIN, G. CALISIR 2008. Distribution of Androctonus crassicauda (Olivier, 1807) and Buthacus macrocentrus (Ehrenberg, 1828) (Scorpiones: Buthidae) in Turkey. Serket, 11(1):13-18.

Citation: Shahrokh Navidpour, "Scorpion Fauna of Qazvin Province, Iran (Arachnida, Scorpiones)" International Journal of Research Studies in Zoology, vol. 6, no. 1, p. 12-19, 2020. DOI: http://dx.doi.org/ 10.20431/2454-941X.0601003.

Copyright: (C) 2020 Authors. This is an open-access article distributed under the terms of the Creative Commons Attribution License, which permits unrestricted use, distribution, and reproduction in any medium, provided the original author and source are credited. 